\begin{abstract}
¿Cómo citar este artículo?
Bermeo-Giraldo, M. C., Acevedo Correa, Y., Palacios Moya, L., Benjumea Arias, M. y Arango-Botero, D. (mayo-agosto, 2020). Evolución y tendencias investigativas sobre estrategias de gestión de conocimiento en instituciones de educación superior. Revista Virtual Universidad Católica del Norte, (60), 202-227. https://www.doi.org/10.35575/rvucn.n60a11
\end{abstract}

\title{
Evolución y tendencias investigativas sobre estrategias de gestión de conocimiento en instituciones de educación superior
}

\author{
Evolution and research trends on knowledge management strategies in Higher \\ Education Institutions
}

\author{
Maria Camila Bermeo-Giraldo \\ Ingeniera Industrial \\ Institución Universitaria Escolme \\ Medellín, Colombia \\ bgmariac@gmail.com, cies2@escolme.edu.co \\ Orcid: https://orcid.org/0000-0001-6501-513X?lang=es
}

\section{Lucía Palacios Moya}

Magister en Salud Pública

Institución Universitaria Escolme

Medellín, Colombia

ciessalud3@escolme.edu.co

Orcid: https://orcid.org/0000-0003-3891-0862

\author{
Yesenia Acevedo Correa \\ Socióloga \\ Fundación Universitaria Católica del Norte \\ Medellín, Colombia \\ yacevedoc@ucn.edu.co \\ Orcid: https://orcid.org/0000-0003-2557-2809
}

\author{
Martha Benjumea Arias \\ Magister en Ingeniería Administrativa \\ Instituto Tecnológico Metropolitano \\ Medellín, Colombia \\ marthabenjumea@itm.edu.co \\ Orcid: https://orcid.org/0000-0002-6776-3892
}

\section{Diana Arango-Botero}

Magister en Estadística

Instituto Tecnológico Metropolitano

Medellín, Colombia

dianaarangob@itm.edu.co

Orcid: https://orcid.org/0000-0002-5184-943X

Recibido: 31 de enero de 2019 Evaluado: 9 de marzo de 2020

Aprobado: 4 de mayo de 2020

Tipo de artículo: Investigación Científica y Tecnológica

\section{Resumen}

Actualmente las instituciones de educación superior -IES- están interesadas en desarrollar y aplicar prácticas o estrategias de gestión de conocimiento que sean efectivas en su interior, ya que estas tienen la misión de ser escenarios de generación y transferencia de conocimiento, en razón a que dinamizan las diferentes dimensiones (social, económica y personal) que componen la sociedad. Este trabajo tuvo como objetivo determinar la evolución y tendencias inmersas en la literatura sobre estrategias de gestión de conocimiento en las IES, presentando los resultados del desarrollo de un estudio bibliométrico, basado en el análisis de la productividad, los autores más representativos, la evolución y las tendencias acerca de la temática propuesta; para la búsqueda de las fuentes bibliográficas se tomó la base de datos de Scopus. Entre los hallazgos, se perfilan como nuevas tendencias: estrategias y herramientas para la formación en línea y la 
utilización de las redes sociales para la gestión y difusión de la información en las IES. Adicionalmente, la revisión de literatura realizada soporta que hay un mejor desempeño en la productividad de la investigación y la eficiencia de los procesos administrativos, mediante la implementación de modelos de gestión de conocimiento en estas organizaciones.

Palabras clave: Análisis bibliométrico; Estrategias; Gestión de conocimiento; Instituciones de educación superior.

\section{Abstract}

There is a growing interest behalf of Higher Education Institutions (HEIs) to develop and apply knowledge management practices or strategies that are effective within these organizations, since these have the mission of being scenarios of knowledge generation and transfer, because they dynamize the different dimensions (social, economic and personal) that make up society. This research aims to determine the evolution and trends immersed in the literature on knowledge management strategies in HEls, presenting the results of the development of a bibliometric study based on the analysis of productivity, the most representative authors, the evolution and the tendencies about the proposed theme. The designed methodology analyzes the selected documents, obtained by means of a search equation in the Scopus databases and calculates the bibliometric indicators of quantity and quality. Among the findings, they are emerging as new trends: strategies and tools for online training and the use of social networks for the management and dissemination of information in HEls. Also, the literature shows that there is better performance in research productivity and efficiency of administrative processes, through the implementation of knowledge management models in these organizations.

Palabras clave: Bibliometric analysis; Strategies; Knowledge management; Higher Education Institutions.

\section{Introducción}

El interés por la gestión del conocimiento se ha vuelto un foco en diferentes espacios organizacionales, debido a que esta plantea la administración, circulación y apropiación del conocimiento que alli se genera, con la intención de elevar sus niveles de competitividad en el entorno (Agudelo y Valencia-Arias, 2018). Así las cosas, la mejora de estas competencias en las universidades permite orientar la gestión del conocimiento hacia la innovación tecnológica, el uso de tecnologías de información -TIC- y demás contribuciones sobre desarrollo científico (Fidalgo-Blanco, Sein-Echaluce \& García-Peñalvo, 2014; Milla, Martelo y Peña, 2018; Velázquez-Juárez, Valencia-Pérez y Peña-Aguilar, 2016). En ese sentido, las instituciones de educación superior -IES- han despertado preocupación por gestionar el conocimiento, ya que estas tienen la misión de ser escenarios de la generación de este, en razón a que dinamizan las diferentes dimensiones que componen la sociedad (social, económica y personal), por medio de la investigación, innovación y creatividad. Ello, posibilita a la universidad la transmisión, distribución y producción del conocimiento, lo que le permite generar agentes de cambio y 
relacionarse permanentemente con otras instituciones (UNESCO, 2009).

Sin embargo, para lograr su gestión, las IES se valen de distintas herramientas que facilitan el almacenamiento en repositorios, puesto que el conocimiento suele usarse para resolver inconvenientes que surgen en el día a día, y no se manifiesta si el mismo puede reutilizarse o debe ser transferido, ocasionando que se necesiten mayores esfuerzos para la realización de búsquedas (Fidalgo-Blanco et al., 2014); además, la mayoría de estas instituciones no tienen registrado ni su conocimiento ni su capital intelectual (Flores, 2010). Lo anterior ocasiona una baja eficiencia ya que la información susceptible de ser transferida se le da a sujetos específicos, lo que retarda la identificación de lo que se necesita (Fidalgo-Blanco et al., 2014). Por ese motivo, se hace necesario que el conocimiento que se produce en ese escenario académico se apropie y transite dentro de las IES. De manera que, la gestión del conocimiento se basa en la estructura de la organización para identificar elementos que faciliten su acceso y correcta administración (Asma \& Abdellatif, 2016).

La gestión del conocimiento en las organizaciones como las IES implica convertir información en conocimiento (Calderón, 2017), lo cual trae como consecuencia el aumento del volumen de la producción de conocimiento organizacional (Fidalgo-Blanco et al., 2014). Por ende, se cree en la construcción de modelos de gestión del conocimiento como una estrategia que refleja la aplicación del conocimiento, planteando una ruta para abordar lo teórico y aplicarlo en lo empírico (Acevedo-Correa, Aristizábal-Botero, Valencia-Arias y Bran-Piedrahita, 2020), en miras de transformar la realidad de la institución implicada, "al explicar la función y ejecución de la gestión del conocimiento en el plano teórico y su aplicación” (Díaz \& Macías, 2013, p. 317).

De acuerdo con lo expuesto anteriormente, este artículo buscó exponer los resultados de un análisis de la literatura desde un enfoque bibliométrico, en el campo de la gestión de conocimiento en IES, que pueda orientar a la construcción de estrategias y prácticas, ya que la intención es analizar la evolución y tendencias que puedan servir para fundamentar propuestas en estos contextos, entendiéndose para esta investigación el término tendencia, como preferencias investigativas o temáticas de estudios abordados, hasta la fecha, por otros autores en la literatura, respondiendo así a los interrogantes: ¿Cómo ha evolucionado la temática en torno a la implementación de la gestión de conocimiento en las IES? y ¿cuál o cuáles son las tendencias en cuanto a la utilización de estrategias de gestión del conocimiento en IES?

\section{Antecedentes sobre la gestión de conocimiento}

Las instituciones modernas, incluidos los establecimientos de educación superior, han visto que cada vez se incrementa de manera exponencial la cantidad de datos derivados de sus procesos cotidianos, los instrumentos en red empleados para albergar los sistemas y variadas aplicaciones necesarias en la ejecución de su labor. Por tanto, estos componentes deben ser administrados y vigilados para su apropiado funcionamiento, al igual que factores fundamentales en la conversión de la información y los conocimientos obtenidos como soporte para la toma de decisiones (Bessa, Branco, Costa, Gonçalves \& Moreira, 2018).

Puede afirmarse que la aplicación del aprendizaje constante y la adecuada gestión del conocimiento, son inevitables en las organizaciones que pretendan suministrar servicios de calidad a sus usuarios, acrecentar el desempeño en la preparación, el aumento de la competiti- 
vidad y adquirir reconocimiento en el contexto global. Por ende, es de vital importancia que estos evalúen la implementación del aprendizaje, y lleven a cabo planes de control y revisión estratégica de su evolución, permitiéndoles dar rápida solución a las demandas de sus clientes, mejorando ostensiblemente el rendimiento de la institución y el logro de propósitos establecidos en los escenarios digitalizados de hoy (Kasemsap, 2015; Londoño-Patiño y Acevedo-Álvarez, 2018).

Hay que mencionar que las IES están llamadas a mejorar la gestión del conocimiento, promoviendo el uso, generación, transferencia e intercambio de conocimiento colectivo; también, fomentar la gestión del conocimiento como parte de la cultura y proyección de la organización, donde se aprecien las capacidades, formación, habilidad para trabajar en equipo y el potencial de aprendizaje profesional (Al-Arimi, Masrom \& Mahmood, 2016). Se debe agregar que es necesaria la adecuación de las tecnologías donde el personal pueda cooperar, apropiar y rescatar conocimiento; al igual que la vigilancia del entorno competitivo, permitiendo la identificación y generación de las oportunidades de proyectos en alianza con distintos grupos de interés (Acevedo-Prins y Jiménez-Gómez, 2015). Por otro lado, la gestión del conocimiento en las IES debería enfocarse en la creación de nuevos servicios, procedimientos, iniciativas prácticas, transferencia y absorción del conocimiento institucional (Al-Arimi et al., 2016). De igual forma, Araya-Guzmán, Henríquez, Ramírez-Correa y Barra (2019) argumentan que las IES deben buscar vincularse a redes de colaboración con fuentes externas (otras instituciones) para apropiarse de nuevos conocimientos y actualizar los existentes, ello garantizaría una utilización eficaz del conocimiento.

No obstante, a los esfuerzos de muchas IES, aún la aceptación del valor de la gestión del conocimiento es reducida, comparado con su evolución en el campo de las compañías y la industria (Alenezi \& Emmanouilidis, 2017). En relación con el tema, la gestión del conocimiento se desarrolla de forma irreflexiva o rutinaria, más no se hace una articulación deliberada y sistemática de los elementos constitutivos del sistema (personas, procesos y actividades) (Morgan \& Llinàs, 2017).

Por su parte, se ha posicionado como un instrumento destacado en la transferencia de conocimiento tácito (basado en experiencias de los individuos, de carácter subjetivo y compleja explicación y materialización), la práctica de crear historias de forma colaborativa, facilitando la captura de este tipo de conocimiento, apoyándose en medios tecnológicos y su posterior transformación en explícito (basado en la formalización del conocimiento a través de informes, mapas, fórmulas), para su difusión a través de narraciones; lo anterior, es conocido bajo el nombre de storytelling, según su denominación en inglés, siendo uno de los principales retos en los procesos de gestión del conocimiento en las IES (Gouvêa, Pimentel, Santoro \& Cappelli, 2016). 


\section{Modelos y sistemas de gestión de conocimiento}

Frecuentemente en la literatura aparecen dos conceptos ligados a la temática de gestión de conocimiento: sistema y modelo. Mohammed (2015) observa que los modelos de conocimiento son una parte integral de las organizaciones que tienen el deseo de establecer sistemas de gestión del conocimiento.

Los sistemas de gestión de conocimiento (KMS, por sus siglas en inglés) se utilizan en la actualidad en prácticamente todos los sectores importantes de la economía mundial (Khabarov \& Volegzhanina, 2019). Estos sistemas constituyen un enfoque integrado que reúne y usa los flujos de información de una organización, mejorando el conocimiento y experiencia de sus miembros (Yankovskaya \& Kukushkin, 2019). Por ejemplo, a través de los KMS las IES gestionan el proceso de adquirir, compartir y crear conocimiento; además de promover el aprendizaje organizativo (Yigzaw, Jormanainen \& Tukiainen, 2019). También, en el estudio de Atanasova (2019), se presenta un KMS que se propuso en una universidad con el objetivo de apoyar la toma de decisiones para el logro de la competitividad y el desarrollo sostenible en algunos procesos relacionados con el aprendizaje a distancia.

Respecto a los modelos de gestión de conocimiento y su aplicación en las IES, varios autores han desarrollado propuestas para crear tales modelos. Por ejemplo, Bocangel Weydert, Bocangel Marin y Pastrana Díaz (2019), para proponer un modelo de gestión, parten de la revisión de los siguientes modelos: (a) Modelo de KPMG Consulting, (b) Modelo Skandia, (c) Modelo Technology Broker, (d) Modelo del Canadian Imperial Bank, (e) Modelo de dirección estratégica por competencias, y (f) Modelo de gestión del conocimiento-Arthur Andersen. Por otra parte, Hansen, Mork \& Welo (2019) tomaron como referencia el modelo de Nonaka y Takeuchi, en aras de proponer un modelo conceptual de gestión de conocimiento para la colaboración universidad-industria, en cuanto a proyectos de innovación. Otro de los modelos de gestión de conocimiento que ha servido como base para la construcción y propuesta de un modelo, en el contexto de las instituciones de educación, es el de INTELEC, el cual fue utilizado en la investigación de Correa, Rosero y Segura (2008). Budiastuti \& Prabowo (2017), por su parte, construyeron un modelo de gestión de conocimiento en universidades privadas, en donde se tuvieron en cuenta aspectos como el liderazgo, los recursos humanos, la transformación de procesos y las TIC.

\section{Importancia gestión de conocimiento en IES}

Tomando como referente a De Freitas y Yáber (2015), el objetivo fundamental de la gestión de conocimiento es aprehender todo el conocimiento que surge del relacionamiento entre los integrantes de la institución y situarlo al alcance de estos, con el fin de robustecer su factor diferenciador y proporcionar soluciones reales, apoyadas en sus capacidades, a quienes son su razón de ser. Además, para su implementación se requiere de elementos como el software y el hardware, concretamente sustentados en plataformas tecnológicas de conocimiento, basados en una estructura flexible a las novedades tecnológicas, y, aún más importante, soportados por estrategias de inteligencia en lo formativo, desde la contribución a la educación, el aprendizaje, la exploración científica (investigación), depósitos de datos, entre otros. Así, De Freitas y Yáber (2015) proponen un diseño enfocado en los principales procesos ligados a la gestión del conocimiento (caracterización, recolección, organización, depósito, recuperación, aplicación y entrega de saberes), enfatizados en los procesos estratégicos de las instituciones de formación 
del nivel superior, con el fin de ayudar en la integración de diversas áreas de gestión; este es un modelo que permite la adaptación y combinación de tecnologías de información y comunicación -TIC- presentes, y la inclusión de nuevas.

El proceso debe incluir, de manera consistente, el intercambio de información que favorezca tanto a las personas como a las instituciones. Además, tomando en consideración la influencia de los sistemas de gestión del conocimiento utilizados por las IES, es importante resaltar que la incorporación de TIC facilita la acumulación de datos, asociados al desempeño analizado a partir de indicadores, específicamente cuando lo que se mide es la calidad en la parte científica (Fernández-López, Rodeiro-Pazos, Calvo \& Rodríguez-Gulías, 2018). Al respecto, conviene decir que las IES de hoy, para dar una mejor respuesta a inquietudes que surgen de los procesos que involucran sus actividades de formación, deben incorporar herramientas de minería de datos, dados los grandes volúmenes de información que manejan; esto como mecanismo valioso en la gestión del conocimiento (Natek \& Zwilling, 2014). En tal sentido, se ha de tener en cuenta la trascendencia de la recopilación y análisis de los datos, estructuración, valoración, distribución y protección de la información, y conocimientos como requisito esencial de su gestión (Roohbakhsh, Gholizadeh \& Javidi, 2017).

Además, estas organizaciones sustentan sus sistemas de gestión del conocimiento en aspectos como la proyección sistemática y la planificación estratégica de las acciones más relevantes ejecutadas, mediante la integración y ordenación de los distintos componentes del proceso, de acuerdo con su nivel de importancia, promoviendo el crecimiento en todas las áreas (institucional, académico, investigativo y tecnológico). Este tipo de estructuración del conocimiento está relacionado con una orientación en espiral, donde se exhibe el continuo e interminable avance entre las diversas etapas y actividades, lo que a su vez genera mejoras por medio de un proceso reiterado e incremental, puesto que es posible la adición de nuevos proyectos y operaciones, en aras de la consecución de las metas propuestas (V. H. Medina, L. M. Medina \& Meza, 2018).

De cualquier manera, para Hansen, Mork \& Welo (2017) el avance rápido de las tecnologías y cambio acelerado de las economías reta a las instituciones a crear estructuras capaces de estimular constantes dinámicas de aprendizaje e innovación. Para ello, debe gestionarse el conocimiento adecuadamente, a partir del fomento de la participación en proyectos innovadores, donde se vinculen las universidades y las empresas, propiciando un impacto significativo de la innovación, en beneficio de las organizaciones y la sociedad; lo anterior, teniendo en cuenta que, para lograr el éxito en la gestión del conocimiento, la mayoría de las IES se han enfocado en el desarrollo y utilización de plataformas digitales, preocupadas por el intercambio de información, restándole importancia al talento humano (Muñoz \& Valencia, 2015). Sin embargo, se debe establecer y fortalecer una estrategia interna de comunicación, dinamizadora de integración de las personas, promoción del empoderamiento e incorporación de principios éticos en la cultura de las entidades; de la misma manera, estimular la externalización, participación y creación de conocimiento en las IES (Muñoz \& Valencia, 2015).

Dentro de este contexto es necesario resaltar que existen elementos asociados con los individuos, como los principios éticos, las capacidades, el profesionalismo y la inspiración, los cuales pueden ejercer un impacto positivo en su rendimiento, lo que obliga a las instituciones a mejorar en este componente en particular (personas) para el incremento en su desempeño 
(Suroso, Sasmoko \& Elisabeth, 2017). Hay que advertir que, según el criterio de Morgan \& Llinàs (2017), entre los elementos determinantes en la gestión efectiva del conocimiento, en el entorno académico, están: el talento humano idóneo, con gran sentido de pertenencia y compromiso investigativo; al igual que el interés de los directivos, promoviendo e institucionalizando una cultura de construcción, intercambio y utilización del conocimiento; así como la disposición y capacidad de aprovechamiento del aprendizaje.

Ahora bien, Astuti, Pratolo \& Anwar (2017) afirman que los dirigentes universitarios deben estar en capacidad de estimular el perfeccionamiento de destrezas, conocimientos y actitudes en el personal que contribuye al logro de propósitos organizacionales (docentes, personal administrativo y estudiantes). En ese sentido, algunos estudios indican que es importante la participación de los docentes en el desarrollo de la gestión del conocimiento; a pesar de eso, se afirma que la generación de conocimiento es derivada esencialmente de los trabajos en niveles de posgrado (maestrías y doctorados), esto confrontado con indicadores de resultados sobre las publicaciones efectuadas en revistas indexadas (Oktavia, Spits, Adi, Prabowo \& Supangkat, 2017).

De otro lado, en la exploración de alternativas para el progreso continuo de la educación superior en Colombia, se está optando por la implementación de sistemas de apoyo que permitan optimizar los procesos, como es el caso del CHE2A (herramienta que facilita la gestión de la formación superior como servicio público), donde los diferentes partícipes pueden, entre otras: dilucidar, estimar, discutir, proponer, establecer, diseñar, planificar, aplicar y evaluar estrategias, condiciones y operaciones definitivas en el desarrollo, mejora e innovación de los programas ofertados por las IES. En síntesis, el CHE2A es un sistema de gestión de conocimiento basado en la experiencia de las organizaciones y orientado a apoyar la innovación en la educación superior, para obtener un servicio con mayor rendimiento (Llamosa-Villalba, Carreño, Paéz, Barajas \& Sneyder, 2015). Desde este ángulo el consolidar la difusión del conocimiento y la cooperación entre entidades del sector educativo incrementa la calidad de la educación, por lo que las universidades deben estar en condiciones de preparar talento humano competente para responder a las exigencias de la sociedad, basadas en el uso de tecnologías emergentes, convirtiéndose en estructuras de categoría mundial; además, apalancadas en sus experiencias y aprendizaje acumulado, logrando mayor eficiencia. Es más, los estudiantes de estas instituciones deben exigirse al máximo e involucrarse en proyectos de investigación científica, logrando con esto mayor calidad en su preparación (Passailaigue, Márquez, Ortega y Febles, 2017).

\section{| Metodología}

Esta investigación planteó una metodología basada en el análisis bibliométrico, que buscó indagar sobre cuáles son los temas tendencia en la implementación de estrategias de gestión de conocimiento en las IES, y cuál es la evolución y estado actual de la temática. La bibliometría o los estudios bibliométricos son utilizados para analizar la información asociada a la producción científica (Thelwall, 2009); hoy en día, también puede aplicarse para la evaluación cuantitativa de la producción académica, con el fin de abordar las tendencias en un área de estudio determinada, mediante el análisis de documentos existentes, como libros, informes, tesis, 
disertaciones, artículos publicados, entre otros (Shi \& Li, 2019). Para la presente investigación, se diseñó un estudio descriptivo de corte longitudinal, con el fin de determinar la evolución o cambios en las publicaciones sobre la gestión de conocimiento en las IES, desde los inicios de la temática hasta la actualidad. El desarrollo de la investigación consistió en tres fases principales, las cuales se presentan resumidas en la figura 1, como el diseño metodológico.

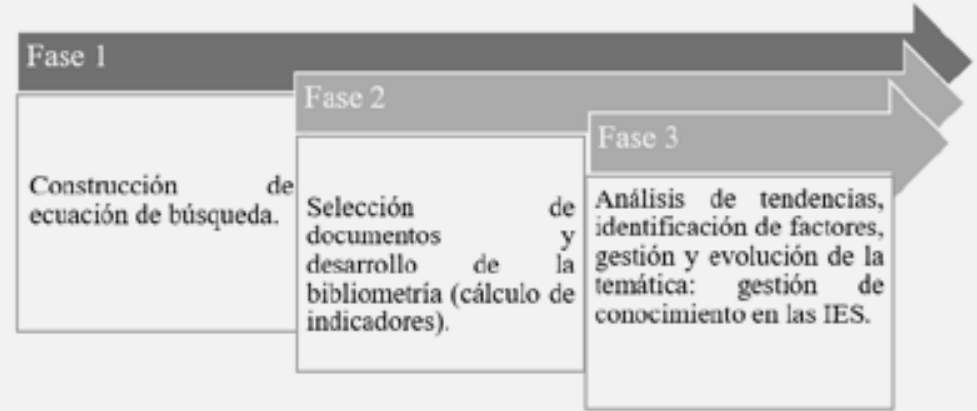

Figura 1. Resumen etapas del análisis bibliométrico. Fuente: adaptado de Thelwall (2009).

Fase 1

La primera etapa de la metodología contempló la construcción de la ecuación de búsqueda de la literatura. Esta se realizó mediante la combinación de los términos clave como: "knowledge management", "model", "system", "University, "higher education" y "college", en la base de datos Scopus, dado que esta es una plataforma muy reconocida con literatura científica en el ámbito internacional. Finalmente, la ecuación consolidada fue: (TITLE (\{Knowledge Management $\}$ ) AND TITLE ( \{model\} OR \{system\}) AND TITLE-ABS-KEY ( universit* OR \{higher education\} OR \{college ) ), y se aplicó en diciembre del 2019.

\section{Fase 2}

En la segunda fase, se consideró la descarga y depuración de los documentos encontrados, a partir de algunos criterios que sirvieron de filtro, como el título, año de publicación, resumen, palabras clave, tipo y fuente del documento, idioma, instituciones (afiliaciones) y país. El uso de Microsoft Excel permitió organizar en columnas cada uno de los registros o documentos arrojados en la búsqueda, y revisar, por medio de filtros, la completitud de los criterios mencionados anteriormente. En esta sección se realizó una rigurosa revisión de los registros encontrados para clasificarlos y proceder al cálculo de los indicadores.

\section{Fase 3}

Para la etapa de procesamiento de la información de los artículos seleccionados se calcularon una serie de indicadores mediante Excel. De acuerdo con Rehn, Gornitski, Larsson \& Wadskog (2014) un indicador es una variable cuantitativa o cualitativa que proporciona una base sencilla y 
fiable para evaluar los logros, los cambios o la actuación. Entre estos indicadores bibliométricos se tienen los indicadores de cantidad y calidad; los primeros dan información sobre el número de publicaciones producidas por una unidad de investigación (por ejemplo, cantidad de publicaciones por año, por revista, área, entre otros); y los segundos proporcionan información sobre el número de citas que han recibido las publicaciones (por ejemplo, número de citaciones por autor, por revista, entre otros) (Waltman \& Noyons, 2019).

\section{| Resultados}

\section{Indicadores de cantidad}

Luego de procesar los registros encontrados, se identificaron en total 177 documentos entre los años 1999 y 2020, que en general demuestran un creciente interés en el tema. Inicialmente, se destaca el año 2006 con 8 publicaciones relacionadas con casos de estudio para la implementación de los pasos de la gestión de conocimiento (capturar, almacenar, compartir, aprender, explorar y explotar el conocimiento), y la mejora de la competitividad universitaria a través de la gestión de conocimiento- GC, basada en el uso de TIC. Para el año siguiente se observa que el número de publicaciones disminuye a 5, para aumentar nuevamente hasta el 2010 con 11 documentos, resaltándose, entre los estudios, temas como el desarrollo de estructura y evaluación del desempeño de sistema de gestión del conocimiento, vinculación de la GC con redes sociales y la GC como estrategia de reingeniería de la educación superior. De nuevo, entre el año 2011 y 2019 se observa, de forma alternada, incrementos y decrecimientos constantes, destacándose el año 2017 como el año más productivo con 23 publicaciones, tal cómo se observa en la figura 2. Entre los temas tratados por los investigadores durante el periodo 2017, se destacan: indexación semántica latente en el proceso de recuperación del conocimiento, impacto de la GC en el desempeño de la educación superior (formación), innovación del repositorio institucional en universidades, metodología e implementación de sistema de GC en el área de tesorería de escuela politécnica superior (desempeño administrativo), ente otros.

Posteriormente, se evidenciará cómo la tendencia de investigación en el tema se ha centrado en el desarrollo de herramientas y prácticas de GC, especificándose en estudios de IES y para la formación en e-learning. 


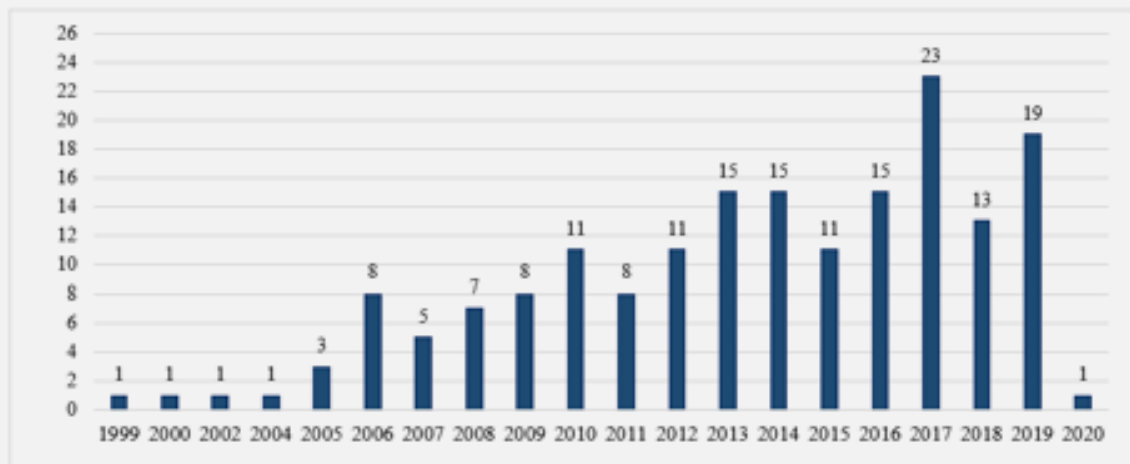

Figura 2. Número de publicaciones por año. Fuente: elaboración propia basada en resultados de Scopus.

A continuación, se presentan las primeras 11 revistas y conference paper como principales fuentes de información (las que más publican acerca del tema), encargadas de publicar los 177 registros asociados a los artículos referentes a la GC en las IES. En consecuencia, el primer lugar lo ocupa Advances in Intelligent Systems and Computing, con 7 publicaciones. Esta revista publica principalmente conferencias y congresos sobre teoría, aplicaciones y métodos de diseño de sistemas inteligentes e informática, principalmente en áreas temáticas como la inteligencia computacional, computación blanda (redes neuronales), sistemas difusos, computación evolutiva e inteligencia social, ambiental, neurociencia computacional, vida artificial, sociedad, ciencia y sistemas cognitivos, percepción y visión, ADN y sistemas autoorganizados y adaptativos. En otras palabras, todas las herramientas informáticas utlizadas para el establecimiento de los modelos y sistemas de gestión de conocimiento.

Se ubica, en segundo lugar, International Proceedings of the European Conference on Knowledge Management-ECKM, con 6 publicaciones, compilando las investigaciones de conferencias y reuniendo papers académicos, estudios de caso, documentos de doctorado y contribuciones no académicas o profesionales. Además, ofrece espacio de discusión de temáticas relacionadas con la gestión del conocimiento en la organización y en la práctica, innovación de conocimiento, capital intelectual, sistemas de gestión del conocimiento, intercambio y economía de conocimiento (Chen \& Lin, 2006).

En el tercer lugar se encuentra, Communications in Computer and Information Science- CCIS, con 5 publicaciones, la cual publica principalmente papers de conferencias, y su objetivo es divulgar eficientemente, de forma impresa y electrónica, resultados de investigaciones originales, relacionados con la informática. Los temas de la CCIS abarcan las concepciones fundamentales en la teoría de la informática, hasta la ciencia y tecnología de la información y las comunicaciones, y variados campos de aplicación interdisciplinaria. Estas publicaciones sólo son recibidas en idioma inglés, lo que garantiza la difusión del tema en el contexto internacional (Palgrave, 2018). 
El cuarto lugar lo comparten las siguientes revistas: Journal of Civil Engineering and Management Lecture Notes in Electrical Engineering, VINE, Journal of Information and Knowledge Management Systems, Journal of Physics: Conference Series y Lecture Notes in Electrical Engineering, con 3 documentos cada una. Para ejemplificar, la revista Journal of Civil Engineering and Management publica investigaciones originales, proporcionando información importante y nuevas ideas, con el fin de mejorar la competencia, la eficiencia y la productividad de la ingeniería civil en los mercados mundiales. Entre las áreas relacionadas a la gestión del conocimiento se encuentra la utilización de tecnologías de información en la construcción y el desarrollo de tecnologías; las publicaciones de VINE, ahora denominada VINE Journal of Information and Knowledge Management Systems (Emerald Publishing, s.f.), son artículos, opiniones y estudios de casos relacionados con la información, desde la perspectiva de sistemas de gestión de conocimiento y la bibliotecología. Las publicaciones de Lecture Notes in Electrical Engineering corresponden a últimos desarrollos de investigación, en todas las áreas de la informática y las nuevas perspectivas en sus campos clásicos, con altos estándares de excelencia académica (Springer, s.f.). El listado de las demás revistas con el mayor número de pulicaciones en el campo de estudio se observa en la figura 3.

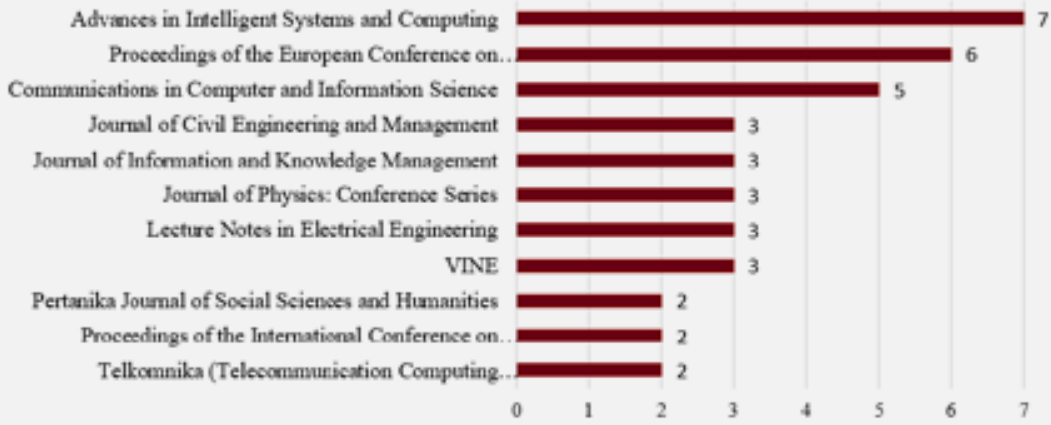

Figura 3. Cantidad de publicaciones por revista. Fuente: elaboración propia basada en resultados de Scopus.

Continuando con el análisis, la figura 4 presenta los diez autores que tienen la mayor producción en el área de estudio. Encabezando el listado se ubica la investigadora Rose Alinda Alias (Alias, R.A.), quien ha publicado 4 documentos sobre la temática; es profesora adscrita al Departamento de Sistema de Información en la Universiti Teknologi Malaysia, y su área de investigación se centra en sistemas de información (informática empresarial), gestión del conocimiento y sociedad digital; su proyecto más reciente es "Educación superior en la cuarta revolución industrial". También, en esta misma posición, se ubica el investigador Fattah Nazem, quien ha publicado 4 documentos sobre la temática; él es profesor adscrito al Departamento de Educación en Roudehen Branch de la Islamic Azad University, Roudehen de Irán, y su área de investigación es el desarrollo de modelos de gestión de conocimiento aplicados en la educación; además, es doctor en gestión de educación y autor de artículos y publicaciones sobre modelos estructurales para el rendimiento en las universidades, basado en la gestión del conocimiento y modelo de ecuación estructural de la gestión del conocimiento para el empoderamiento en las 
IES. Los siguientes autores en la lista son Rahman A.A., Warnars H.L.H.S., Adi S., con 3 publicaciones cada uno; y luego, Bhiriyawanit C., Borisevich V., Asma K., Chakpitak N., y Abdellatif M., con 2 documentos cada uno.

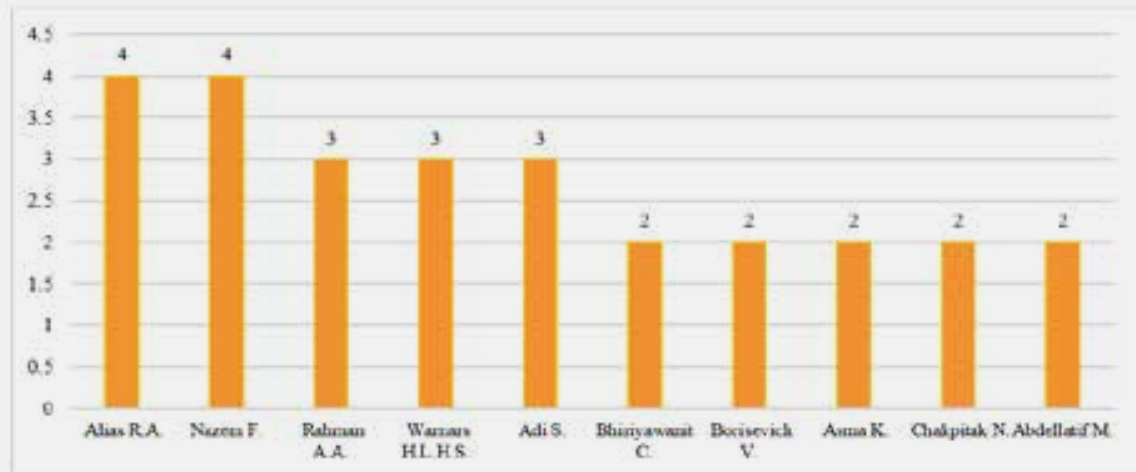

Figura 4. Autor con la mayor producción. Fuente: elaboración propia basada en resultados de Scopus.

El análisis de publicaciones por áreas temáticas en la que se han desarrollado las investigaciones se observa en la figura 5. Los resultados indican que el mayor porcentaje de publicaciones son del área de Computer Science, con el 26\%; seguido de Social Sciences, con 18\%, Engineering, con 16\%; y Business, Management and Accounting con 14\%.

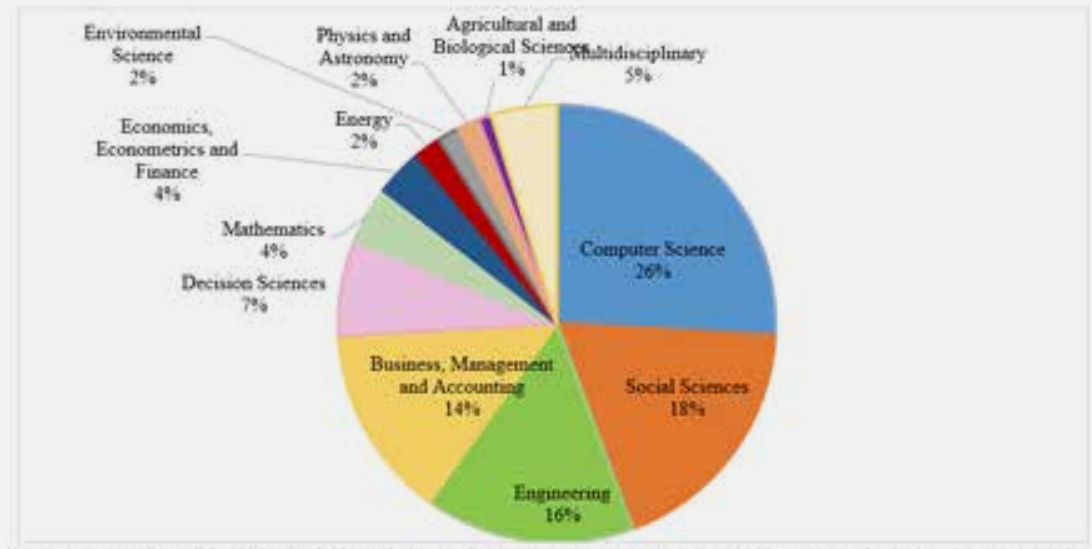

Figura 5. Cantidad de publicaciones por área. Fuente: elaboración propia basada en resultados de Scopus. 


\section{Indicadores de calidad}

Para medir el desempeño de los resultados de las investigaciones, se analizan los indicadores bibliométricos de calidad, a partir del número de citas, lo cual permite conocer el impacto de los trabajos publicados por revista, autor y año (Marulanda-Valencia y Valencia-Arias, 2019).

En la figura 6 se presenta el impacto o cantidad de citaciones por autor, de los diez con mayor cantidad de citaciones en el tema. En primer lugar, Zwilling M. y Natek S., se encuentran con 85 citas; son autores del artículo "Student data mining solution-knowledge management system related to higher education institutions", el cual documenta un estudio sobre el análisis de información, obtenido mediante la minería de datos, para la predicción de la tasa de éxito de los estudiantes inscritos en un curso académico, concluyendo que esta herramienta de gestión de conocimiento es útil por la identificación de patrones de comportamiento y para los tomadores de decisiones de educación superior; además, de ser fácil de utilizar por el personal administrativo de las IES.

Siguiendo la lista, están los investigadores McAdam R., Moffett S., y Parkinson S., con 63 citaciones; su publicación es del año 2002, denominada "Developing a model for technology and cultural factors in knowledge management: A factor analysis", la cual expone el desarrollo de un modelo de GC, como ejemplo de una aplicación práctica, que puede utilizarse en un amplio espectro de organizaciones, como las universidades, para mostrar cómo los aspectos culturales y tecnológicos de la Gestión del Conocimiento pueden integrarse sistemáticamente en las organizaciones.

Los siguientes investigadores, dentro de los diez primeros en la lista, son Brewer K. y Brewer P., con 58 citas por su artículo: "Knowledge Management, Human Resource Management, and Higher Education: A Theoretical Model". Este documento presenta el desarrollo de un modelo teórico que relaciona la gestión del conocimiento, la gestión de los recursos humanos y los objetivos típicos de aprendizaje del conocimiento, de un programa de educación empresarial acreditado, utilizando la taxonomía de Bloom para clasificar, escribir y medir los objetivos de aprendizaje en los estudiantes (Brewer \& Brewer, 2010).

Luego, se ubican en la cuarta posición: Don R., Greenfield P. y Liyanage S. con 55 citas, de un artículo del año 1999: "Towards a fourth generation R\&D management model-research networks in knowledge management". La metodología de su artículo correspondió a un estudio de caso sobre las prácticas de gestión de la investigación empleadas, por grupos de biotecnología y de investigación farmacéutica, en industrias y universidades, con el fin de describir un enfoque de cuarta generación para la gestión de la investigación. Su propósito consistió en la descripción del concepto de gestión de conocimiento como la generación de capital intelectual, mediante el cual se impulsan negocios futuros y nuevos productos (Liyanage, Greenfield \& Don, 1999).

Finalmente, se analiza si existe coincidencia entre los autores más citados (indicador número de citaciones por autor en la figura 6) y los que más publican (autor con la mayor producción en la figura 4). Los resultados indican que no hay similitud entre el indicador de calidad con el de cantidad, porque ninguno de los 10 autores con el mayor número de citas forma parte de los 10 autores con el mayor número de publicaciones. 


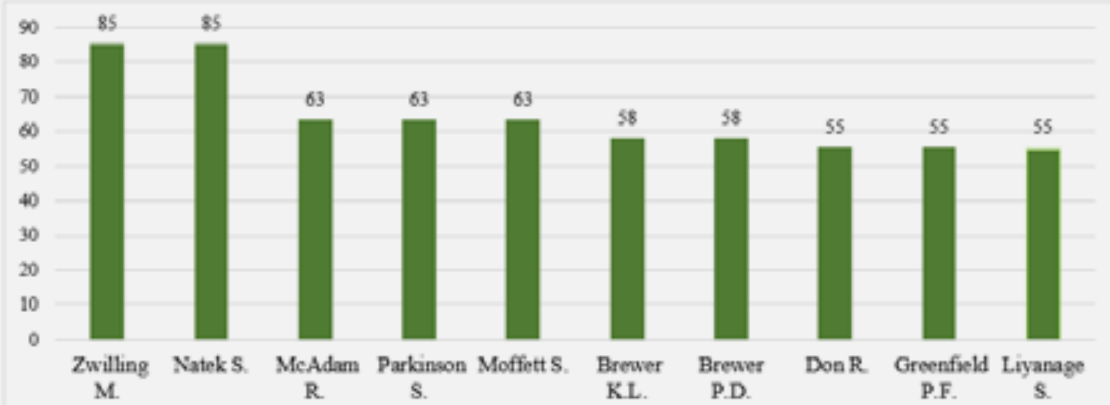

Figura 6. Número de citaciones por autor. Fuente: elaboración propia basada en resultados de Scopus.

En la figura 7 se observa que la revista con el mayor número de citas es Expert Systems with Applications, con 85 citaciones. El propósito de esta revista es aportar documentos relacionados con el diseño, desarrollo, prueba, implementación y gestión de sistemas expertos e inteligentes, y proporcionar pautas prácticas en el desarrollo y gestión de estos sistemas de información. Asimismo, busca divulgar con sus publicaciones documentos sobre sistemas de múltiples agentes, gestión del conocimiento, redes neuronales, descubrimiento de conocimiento, minería de datos, minería multimedia y algoritmos genéticos, promoviendo el intercambio entre sistemas expertos e inteligentes, analizados por investigadores de diversas disciplinas (ELSEVIER, 2020).

La segunda revista con el mayor impacto es Knowledge and Process Management, con 63 citaciones; esta revista tiene como objetivo suministrar información esencial para ejecutivos encargados de mejorar el rendimiento en sus negocios o de introducir nuevas ideas en los negocios, a través del liderazgo intelectual. También, provee información práctica sobre las lecciones aprendidas de otras organizaciones en las áreas de: conocimiento administrativo, aprendizaje organizacional, competencias básicas y gestión de procesos. Entre los tipos de investigaciones, la revista publica estudios de caso detallados, artículos de investigación que contienen nuevas ideas de las principales escuelas de negocios, y trabajos prácticos de encuestas entre empresas (Inderscience Publishers, s.f.).

Luego, la revista que ocupa el tercer lugar con el mayor número de citaciones es Journal of Education for Business, con 58 citas. Esta revista está orientada para educadores de las áreas de negocios y se enfoca en la investigación de temas comerciales tradicionales y de emprendimiento, presentando principalmente artículos basados en investigación básica y aplicada, en emprendimiento, contabilidad, comunicaciones, economía, finanzas, sistemas de información, administración, mercadotecnia y otras disciplinas comerciales. Las publicaciones informan innovaciones exitosas en la enseñanza y el desarrollo curricular, en el contexto universitario y de posgrado. 
De las 10 revistas con el mayor número de citas sobre la temática, el $45.45 \%$ pertenecen a Scopus Q2, 36.36\% a Q3, 9.09\% a Q1, y aproximadamente el 9.09\% no están clasificadas en el cuartil (índice de alto impacto según Scopus), por ser un documento de conferencia o evento académico (ver figura 7). De lo anterior, se tiene que las revistas de mayor calidad no son las que poseen un mayor número de citas en el tema; sin embargo, las publicaciones más citadas se encuentran por encima de la media en el cuartil Q2. Entre las áreas temáticas publicadas en estas revistas y que se encuentran establecidas por Scopus se identifican negocios, administración, contabilidad, relaciones industriales, estrategia y gestión, ciencias sociales, educación, informática, sistemas de información y comunicación.

Finalmente, al comparar el indicador de calidad que mide el impacto en citaciones (ver figura 7) con el indicador de cantidad de publicaciones por revista (ver figura 3), se encuentra que 2 de las 10 revistas analizadas hacen parte de ambos indicadores, Journal of Information and Knowledge Management y Journal of Civil Engineering and Management, lo que evidencia la contribución e impacto existente en la generación del tema tratado y su divulgación en el contexto académico.

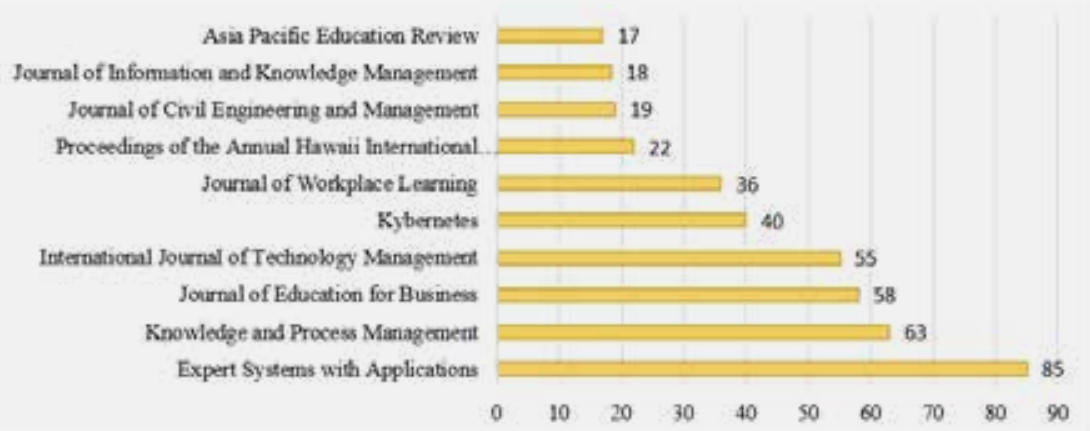

Figura 7. Número de citaciones por revista. Fuente: elaboración propia basada en resultados de Scopus.

\section{| Discusión}

La ley de productividad indica que el conocimiento sobre estrategias y prácticas de gestión de conocimiento en las IES está disperso en varias revistas científicas (no hay revistas académicas que tengan una concentración significativa de estudios sobre el tema), ya que la ley de Pareto no se cumple: el $75.86 \%$ de las revistas publica el $80 \%$ de los estudios sobre gestión de conocimiento en las IES. Luego, se separa el porcentaje de revistas por cuartil (cuatro partes iguales), con el fin de observar las revistas de mayor impacto, analizando la dispersión y la tendencia central de este conjunto de datos, respecto a cuánto publica el 25\% (cuartil 1), cuánto publica el 50\% (cuartil 2) y cuánto publican el 75\% (cuartil 3) de las revistas. En este sentido, por 
ejemplo, el primer cuartil 25\%, corresponde al cuartil de mayor impacto, es decir las revistas con mejor posicionamiento en la temática de estudio. Se obtiene, entonces, que el $9.48 \%$ de las revistas publicaron el $25 \%$ de los artículos, el $39.66 \%$ de las revistas publicaron el $50 \%$ de los artículos; y finalmente el $69.83 \%$ de las revistas publicaron el $75 \%$ de los artículos

En cuanto al autor más productivo se encontró al profesor Nazem F., del Departamento de Educación en Roudehen Branch de la Islamic Azad University en Irán. Su experiencia en investigación incluye el desarrollo de modelos de gestión de conocimiento aplicados en la educación para el rendimiento de las universidades e IES. En el caso de los autores más citados, lo cual refleja el impacto de las publicaciones sobre el tema, están los investigadores McAdam R., Moffett S. y Parkinson S., con 60 citaciones, por su publicación: “Developing a model for technology and cultural factors in knowledge management: A factor analysis", documento más citado que convierte el año 2002 como el de mayor impacto sobre los modelos de GC en las IES. La investigación describe el diseño de un modelo conceptual para la implementación de la gestión del conocimiento, basado en una asociación universidad e industria, utilizando un caso de estudio de doctorado. Los autores concluyen que GC dentro de una organización se caracteriza por elementos técnicos, de información y personales. Aunque el tema se ha desarrollado en algunas organizaciones, su avance es aún pobre, dado que muchas no han logrado un cambio organizacional, utilizando un enfoque orientado al conocimiento (cultura). El enfoque óptimo para la implementación de GC dentro de las organizaciones es la cultura y el uso de tecnologías. Por su parte, la revista de mayor impacto es Knowledge and Process Management con 60 citaciones, la cual provee información práctica sobre las lecciones aprendidas de otras organizaciones en las áreas de: conocimiento administrativo, aprendizaje organizacional, competencias básicas y gestión de procesos.

En una revisión sistemática, los autores Acevedo-Correa, Valencia-Arias, Bran-Piedrahita, Gómez-Molina y Arias-Arciniégas (2019) encuentran que los teóricos que más se destacan son Nonaka y Takeuchi, como principal referente para la creación e interacción de conocimientos tácitos (empíricos) y explícitos (formalizados); y mencionan que aunque este desarrollo se usa en el contexto organizacional, sus preceptos han sido adaptados en lo educativo, enfocándose en la vinculación de herramientas tecnológicas para permitir la circulación del conocimiento desde diversas estrategias de su gestión, aplicándolas de acuerdo a su contexto interno y externo.

Evolución de temáticas abordadas en estrategias de gestión del conocimiento en IES por períodos de publicación

A continuación, se presenta la evolución de las temáticas abordadas dentro de las publicaciones sobre prácticas, herramientas, modelos y estrategias implementadas en la gestión de conocimiento en instituciones universitarias, que fueron revisadas para esta bibliometría.

\section{Periodo 2000 al 2006}

Durante los años 2000 a 2006, se comienza por comprender la organización como un todo orgánico, en la cual se debe estimular el aprendizaje y el compartir el conocimiento, planteando una relación entre lo tecnológico y cultural (Leonard, 2000). En ese sentido, las IES son concebidas como organizaciones educativas o académicas, por lo cual es necesario que la tecnología y demás herramientas y prácticas, que constituyen estrategias de aplicación de la 
gestión del conocimiento, como los modelos, vayan en la misma vía, en aras de lograr la implementación de conocimiento e impactar el papel profesional de los educadores y el currículo universitario (Kleist, Williams \& Peace, 2004).

La parte comunicacional influye en la transferencia de conocimiento, en tanto es la que permite que la información relevante sea transferida efectivamente (Raman, Ryan \& Olfman, 2006); esto se acopla con la implementación de herramientas web, que permiten al personal acceder, almacenar y crear información, con la intención de intercambiar conocimientos y agilizar la toma de decisiones estratégicamente (Chen \& Lin 2006). Aparece el interés por medir el nivel de madurez de la gestión del conocimiento en IES, ya que esta es una herramienta para describir el estatus e indicadores que dan cuenta del desarrollo de la GC como estrategia para mejorar las prácticas investigativas.

\section{Período 2007 al 2012}

Entre el 2007 y 2012 se trajo a colación la importancia de estudiar la GC en IES, ya que contribuye a la generación de conocimiento científico, manteniendo un interés por el proceso comunicacional como eje para el establecimiento, por ejemplo, de modelos de gestión del conocimiento (Lima y De Souza, 2007). Para ello, es relevante aplicar esto, tomando como referente, además, el contexto social que rodea a estas instituciones, de esa manera se tiene una comprensión más orgánica para la aplicación de estos sistemas.

La implementación de modelos de gestión del conocimiento, por medio de aplicaciones web, permiten una mejor forma de manejar información y documentación de proyectos de investigación, estimulando así la colaboración entre científicos, por el nivel de procesamiento y de visualización de los datos necesarios para la estimulación de actividades de investigación (Akhavan, Owlia, Jafari \& Zare, 2011). Dentro de esos aplicativos se tienen la utilización de portafolios digitales recopiladores de programas de estudio, herramientas de devaluación, recibir retroalimentación y realizar auditorías periódicas que garantizan la calidad y estándares de los programas (Maher \& Kourik, 2008). También, se plantea que estos sistemas se implementen para el e-learning como apuesta educativa, con la utilización de foros para intercambios de opinión, repositorios y enlaces con expertos relacionados (Abu-Shawar \& Al-Sadi, 2008).

En las universidades públicas se busca el desarrollo de competencias, a partir de la creación de conocimiento que pueda aplicarse al apoyo de comunidades en las que estas tienen impacto; para ello, se promueven las capacidades de estudiantes respecto al uso de TIC, logrando además servicios innovadores (Numprasertchai \& Poovarawan, 2008). En ese orden, los sistemas de gestión del conocimiento aportan en la misión universitaria de investigación y extensión. Una manera de llevar a cabo lo planteado anteriormente, es por medio de planificación como estrategia para gestionar el conocimiento, basándose en motivaciones y potencial del equipo de trabajo, y así actualizar el cuerpo de conocimiento y evaluación de conclusiones, que aporten al éxito en el liderazgo y administración de actividades en el marco educativo (Chantarasombat, 2009).

Otro aspecto importante es la aplicación de diagnóstico para medir y guiar la implementación de la gestión del conocimiento en organizaciones e instituciones educativas (Teah, Pee \& Kankanhalli, 2006). Lo anterior, con el fin de que se identifiquen los problemas que aparecen en el aprendizaje en línea, y así construir un sistema de gestión del conocimiento basado en compartir recursos educativos y satisfacer las demandas educativas, eficaz y eficientemente. 
Periodo 2013 al 2019.

Para el periodo comprendido entre 2013 al 2019, se resaltan algunos tópicos que son tendencia en el contexto académico, como el e-learning; las universidades aplican las herramientas de gestión de conocimiento y tecnologías en los sistemas de educación superior para enseñar las diferentes áreas de conocimiento. Para la mejora de los planes de estudio, por ejemplo, se suele combinar aprendizaje social y conceptos de gestión del conocimiento en los entornos e-learning de las universidades (Muñoz-García, Del Cioppo \& Bucaram-Leverone, 2017). Al interior de las IES, las bibliotecas administran la información a través de la incorporación de elementos de gestión de conocimiento, lo que significa un cambio de gerencia hacia una más moderna, electrónica, digital y de red. También, funciona como un repositorio para aumentar la producción científica y para solucionar la principal dificultad de las universidades: acceder al conocimiento y centralizarlo, para luego consultarlo y difundirlo o trasmitirlo efectivamente.

De acuerdo con García-Álvarez, Pineiro-Villaverde \& Varela-Candamio (2018) el uso de modelos de gestión del conocimiento en las IES promueve que los estudiantes consigan una mayor comprensión del conocimiento. Lo anterior, es posible gracias a la utilización de páginas web, plataformas en línea y softwares. También, se destaca la utilización de aplicaciones móviles para facilitar el acceso al conocimiento, por parte de alumnos y profesores.

La elaboración de los sistemas de GC, considera como elemento importante la previa clasificación del conocimiento (explícito y tácito) y una clara definición de las funciones de sus componentes. Por otro lado, considerar factores como infraestructura, capital intelectual, el entorno organizacional, procesos comerciales, aprendizaje organizacional, sistema cultural, aprendizaje en equipo; además, la innovación es la base para la gestión eficiente de la aplicación de GC en las universidades.

Otros elementos adicionales a la utilización de las TI, especialmente en la implementación de modelos de GC en las IES, es definir el activo intelectual, como el conocimiento activo; el aprendizaje organizacional como el entorno de la organización que facilita el conocimiento; los procesos, que condicionan el flujo de este; la filosofía y cultura, orientada a la comprensión del activo intelectual; y las personas con capacidades y competencias pertinentes para interactuar en el sistema de GC. Hallazgos indican que la competitividad de una universidad depende directamente del nivel y la madurez de su sistema de GC (Tkachenko, Rogova \& Karlik, 2017).

En cuanto a las metodologías de investigación, en los estudios sobre estrategias de gestión de conocimiento en IES, los casos de estudio, las revisiones sistemáticas de literatura y la elaboración de marcos conceptuales lideran las investigaciones, cuyo objeto de estudio son las IES. Estas últimas implementan los sistemas de GC no solo para la enseñanza de los cursos, sino también para solucionar los requerimientos internos y ayudar a la realización de procesos administrativos y académicos, de manera más efectiva y eficiente en la universidad.

La figura 8, a continuación, permite observar, de manera resumida, la evolución de la temática, considerando la línea de tiempo entre los periodos 2000 al 2006, 2007 al 2012 y del 2013 al 2019. 

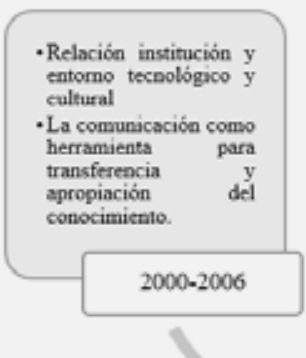

\section{$2007-2012$}

- Conocimiento del contexto social que rodea las IES para implementar estrategias de GC.

- Implementación de aplicativos Web para estimular trabajo colaberativo y estimula la calidad.

- Aplicación de diagnósticos para la generación de estrategias y planes de trabajo de la GC.
- Aplicación de tecnologias relacionadas con elearning para implementar mejorar al curriculo.

- Aparecen las bibliotecas como repesitorios para almacenar $y$ centralizar el conocimiento.

2013-2019

Figura 8. Evolución de la temática, estrategias de GC en las IES. Fuente: elaboración propia basada en resultados de Scopus.

\section{Agenda futura de investigación}

El trabajo futuro propende la utilización de herramientas para minería de datos y minería de textos que hagan más eficientes los procesos de aplicación de GC en las IES, como por ejemplo la detección de tecnologías que se incorporan en los sistemas de gestión de conocimiento para llevar una mejor administración de la información, y poder predecir indicadores importantes en las universidades, como estudiantes matriculados en sus cursos, tecnologías que contribuyen a la generación de innovaciones, tasas de éxito, entre otros.

Por otro lado, se recomienda a futuro profundizar en la utilización de los medios de comunicación social (redes sociales), ya que facilitarían el proceso de intercambio de conocimientos dentro de la universidad; y orientados correctamente pueden apoyar el aprendizaje colaborativo (aprendizaje social) entre los estudiantes. Además, dado que la gestión de conocimiento parte de un proceso social y del capital humano, el estudio de los factores necesarios para la implementación de estos sistemas debe integrar metodologías de investigación cualitativa y cuantitativa.

Del análisis bibliométrico, los resultados indican un crecimiento del tema desde el año 1999 hasta el presente año, y se identifica el año 2017 como el más productivo con 19 publicaciones. De los tipos de publicaciones se evidencia un gran interés por las actas de conferencias, ponencias o comunicaciones en congresos, resaltándose la International Proceedings of the European Conference on Knowledge Management-ECKM, compilando las investigaciones de conferencias, reuniendo papers y planteando espacios de discusión de temáticas de la gestión del conocimiento en la organización, innovación de conocimiento, capital intelectual, sistemas de gestión del conocimiento, intercambio y economía de conocimiento, incluyendo modelos y su aplicación. En adición, a través del análisis bibliométrico de los documentos seleccionados se destaca como método para desarrollar e implementar la GC, la utilización de las TIC en sus diferentes niveles, es decir, desde la generación de aprendizaje y del conocimiento, y la distribución y la transferencia de este. 


\section{| Conclusiones}

La literatura muestra que la aplicación de la gestión del conocimiento contribuye significativamente a mejorar la productividad de la investigación y la eficiencia de los procesos administrativos en las IES, ya que la información que se recoleta no solo es relacionada con el aprendizaje y la enseñanza de las distintas áreas de conocimiento, sino que mediante los sistemas de gestión se administra la información de estudiantes, profesores y demás datos estadísticos del funcionamiento de las IES, con el fin de poder dar respuesta a requerimientos internos, y así mejorar la eficiencia de servicios administrativos: organizar reuniones y aplicar reglamentación y regulaciones en el momento adecuado, para las personas correctas.

De igual forma, se comprende la relevancia adquirida por el e-learning y el aprovechamiento de la inclusión de herramientas electrónicas y los entornos virtuales de aprendizaje, transformando los procesos de formación, difusión y formas de aprendizaje en escenarios cada vez más dinámicos y globalizados, por el uso de las tecnologías de la información y la comunicación; erigiéndose como instrumento vital para la efectividad en el acceso al conocimiento, interacción y trabajo colaborativo entre los diversos participantes. Asimismo, se asimila la importancia de la gestión del conocimiento y el impacto que esta genera en los múltiples procesos desarrollados por las instituciones de enseñanza, como la formación, gestión, investigación e innovación, donde cada vez las universidades deben asumir mayor liderazgo, apalancadas en el aprendizaje e información acumulado, sus procesos y sistemas de gestión del conocimiento, como base crítica en la creación de nuevo conocimiento, desarrollo científico y progreso para la sociedad.

De otro lado, resulta preciso señalar que la gestión del conocimiento presenta temas de interés que deben ser explorados para alcanzar profundidad y entendimiento, ampliando así el aporte científico, con el cual se contribuya al desarrollo de los países emergentes, dada la existencia de diferencias notables en publicaciones e información de patentes; además de enfocarse en auditorías de la gestión del conocimiento, como valor esencial en la implementación de estos modelos; y la investigación focalizada en el rol de las universidades, como ejes impulsadores del avance investigativo e innovación de los países en vías de desarrollo, a través de Su GC y los SGC, alineados de acuerdo con las necesidades específicas de cada nación.

\section{| Referencias}

Abu-Shawar, B. \& Al-Sadi, J. (2008). Knowledge management view extracted from learning management system used at AOU. En Wmsci 2008: 12Th World Multi-Conference on Systemics, Cybernetics and Informatics, Proceedings (pp. 199-205).

Acevedo-Correa, Y., Aristizábal-Botero, C. A., Valencia-Arias, A. y Bran-Piedrahita, L. (2020). Formulación de modelos de gestión del conocimiento aplicados al contexto de instituciones de educación superior. Información tecnológica, 31(1), 103-112. 
Acevedo-Correa, Y., Valencia-Arias, A., Bran-Piedrahita, L., Gómez-Molina, S. y Arias-Arciniégas, C. (2019). Alternativas para modelos de gestión del conocimiento en Instituciones de Educación Superior. Ingeniare, 27(3). http://dx.doi.org/10.4067/S0718-33052019000300410

Acevedo-Prins, N. M. y Jiménez-Gómez, L. M. (2015). Índice para la medición de la competitividad en Colombia. Revista CEA, 1(2), 109-121. https://doi.org/10.22430/24223182.136

Agudelo, E. J. y Valencia-Arias, A. (2018). La gestión del conocimiento, una política organizational para la empresa de hoy. Ingeniare. Ingeniare, 26(4), 673-684.

Akhavan, A., Owlia, M. S., Jafari, M. \& Zare, Y. (2011). A model for linking knowledge management strategies, critical success factors, knowledge management practices and organizational performance; the case of Iranian universities. En 2011 IEEE International Conference on Industrial Engineering and Engineering Management (pp. 1591-1595). https://doi.org/10.1109/IEEM.2011.6118185

Al-Arimi, A. A. A. A., Masrom, M. \& Mahmood, N. H. N. (2016). The moderating effect of Islamic work ethics on the relationship between knowledge management capabilities and organizational performance at the private higher education institutions in Oman. Journal of Theoretical and Applied Information Technology, 94(2), 396-407.

Alenezi, A. \& Emmanouilidis, C. (2017). Utilising learning analytics in knowledge management adoption for effective manufacturing education. En K. S. El Souri M. \& Gao J. (Ed.), 15th International Conference on Manufacturing Research, ICMR 2017 (pp. 534-539). London, England: University of GreenwichLondon. https://doi.org/10.3233/978-1-61499-792-4-534

Araya-Guzmán, S., Henríquez, C., Ramírez-Correa, P. y Barra, A. (2019). Explorando la relación entre gestión del conocimiento y el rendimiento organizativo en Instituciones de Educación Superior Universitaria. RISTI - Revista Iberica de Sistemas e Tecnologias de Informacao, (E17), 947-959.

Asma, K. \& Abdellatif, M. (2016). A New Model for the Impact of Knowledge Management on University Performance. Journal of Information \& Knowledge Management, 15(04). https://doi.org/10.1142/S0219649216500416

Astuti, R. J., Pratolo, S. \& Anwar, M. (2017). College performance based on QS-stars method: Model designing of college performance through knowledge management system (empirical study on a accreditation colleges in Java). International Journal of Applied Business and Economic Research, 15(16), 389-402.

Atanasova, I. (2019). A University Knowledge Management Tool for the Evaluation of the Efficiency and Quality of Learning Resources in Distance e-Learning. International Journal of Knowledge Management (IJKM), 15(4), 38-55.

Bessa, J., Branco, F., Costa, A. R., Gonçalves, R. \& Moreira, F. (2018). Proposal of a BI/SSBI System for Knowledge Management of the Traffic of a Network Infrastructure - A University of Trás-os-Montes e Alto Douro Case Study. En W. 2018 6th World Conference on Information Systems and Technologies (Ed.), Trends and Advances in Information Systems and Technologies (pp. 678-690). https://doi.org/10.1007/978-3-319-77703-0_68 
Bocangel Weydert, G. A., Bocangel Marin G. A. y Pastrana, N. (2019). Un modelo de gestión del conocimiento para las Instituciones de Educación Superior. Opción, 89(2), 573-598

Brewer, P. D. \& Brewer, K. L. (2010). Knowledge Management, Human Resource Management, and Higher Education: A Theoretical Model. Journal of Education for Business, 85(6), 330-335. https://doi.org/10.1080/08832321003604938

Budiastuti, D. \& Prabowo, H. (March, 2017). Quality of Transformation of Knowledge as Part of Knowledge Management System. En International Conference on Information Science and Applications (pp. 827-833). Singapore: Springer.

Calderón, S. (2017). Cultura de investigación y gestión del conocimiento en ciencias sociales. Revista Virtual Universidad Católica del Norte, (50), 343-366. Recuperado de http://revistavirtual.ucn.edu.co/index.php/RevistaUCN/article/view/827/1345

Chantarasombat, C. (2009). Model a knowledge management for educational quality assurance in faculty of education, Mahasarakham university in Thailand. European Journal of Social Sciences, 11(3), 428-440. Recuperado de http://www.scopus.com/inward/record.url?ei$\mathrm{d}=2-\mathrm{s} 2.0-70449556371 \&$ partnerID=40\&md5=c38ec6bede5c26343864bd94ddf7e818

Chen, J. W. \& Lin, C. (2006). A van Hiele Web-based Learning System with Knowledge Management for Teaching Programming. Sixth IEEE International Conference on Advanced Learning Technologies (ICALT'06) (pp. 114-116). https://doi.org/10.1109/ICALT.2006.1652381

Correa, G., Rosero, S. L. y Segura, H. (2008). Diseño de un modelo de gestión del conocimiento para la Escuela Interamericana de Bibliotecología. Revista Interamericana de Bibliotecología, 31(1), 85-108

De Freitas, V. y Yáber, G. (2015). Una propuesta de arquitectura para los Sistemas Informáticos de Gestión del Conocimiento en Instituciones de Educación Superior. Espacios, 36(10), E-2.

Díaz, N. \& Macías, M. E. (2013). Knowledge management model of Center for the Development of Humanities and Social Sciences in Health. Humanidades Médicas, 13(2), 314-329. Recuperado de http://scielo.sld.cu/pdf/hmc/v13n2/hmc03213.pdf

ELSEVIER. (2020). Expert Systems with Applications An International Journal. Recuperado de https://www.journals.elsevier.com/expert-systems-with-applications

Emerald Publishing (s.f.). VINE journal of information and knowledge management systems. Recuperado de http://www.emeraldgrouppublishing.com/products/journals/journals.htm?id=vjikms

Fernández-López, S., Rodeiro-Pazos, D. N., Calvo, N. \& Rodríguez-Gulías, M. J. (2018). The effect of strategic knowledge management on the universities' performance: an empirical approach. Journal of Knowledge Management, 22(3), 567-586.

Fidalgo-Blanco, Á., Sein-Echaluce, M. L. \& García-Peñalvo, F. J. (2014). Knowledge spirals in higher education teaching innovation. International Journal of Knowledge Management, 10(4), 16-37. https://doi.org/10.4018/ijkm.2014100102

Flores, J. C. (2010). La Gestión del conocimiento y las herramientas colaborativas: una alternativa 
de aplicación en Instituciones de educación superior. Revista de Investigación, (71), 11-31., Recuperado de http://ve.scielo.org/scielo.php?script=sci_arttext\&pi$d=S 1010-29142010000300002 \& \operatorname{lng}=e s \& \operatorname{lng}=e s$

García-Álvarez, M. T., Pineiro-Villaverde, G. \& Varela-Candamio, L. (2018). Proposal of a Knowledge Management Model and Virtual Educational Environment in the Degree of Law-Business. En Trends and Advances in Information Systems and Technologies. WorldCIST'18 2018 (pp. 1275-1286). https://doi.org/10.1007/978-3-319-77712-2_122

Gouvêa, M. T. A., Pimentel, M., Santoro, F. M. \& Cappelli, C. (2016). Knowledge management in distance education: An exploratory case study with group storytelling approach. En 11th International Knowledge Management in Organizations Conference on the Changing Face of Knowledge Management Impacting Society, KMO 2016. FernUniversitat. https://doi.org/10.1145/2925995.2926000

Hansen, I.-E., Mork, O. J. \& Welo, T. (2017). Knowledge management of university-industry collaboration in the learning economy. En 2017 2nd International Conference on Knowledge Engineering and Applications, ICKEA 2017 (pp. 173-177).

https://doi.org/10.1109/ICKEA.2017.8169924

Hansen, I. E., Mork, O. J. \& Welo, T. (September, 2019). Managing Knowledge in Manufacturing Industry-University Innovation Projects. En IFIP International Conference on Advances in Production Management Systems (pp. 603-610). Cham: Springer.

Inderscience Publishers. (s.f.). International Journal of Technology Management (IJTM) Inderscience Publishers. Recuperado de http://www.inderscience.com/jhome.php?jcode=ijtm\#moredesc

Kasemsap, K. (2015). The roles of lifelong learning and knowledge management in global higher education. En S. S. R. U. Faculty of Management Sciences (Ed.), Impact of Economic Crisis on Education and the Next-Generation Workforce (pp. 71-100).

https://doi.org/10.4018/978-1-4666-9455-2.ch004

Khabarov, V. \& Volegzhanina, I. (December, 2019). Knowledge management system of an industry-specific research and education complex. En IOP Conference Series: Earth and Environmental Science, 403(1).

Kleist, V.F., Williams, L. \& Peace, A. G. (2004). A Performance Evaluation Framework for a Public University Knowledge Management System. Journal of Computer Information Systems, 44(3), 9-16. Recuperado de https://www.tandfonline.com/doi/abs/10.1080/08874417.2004.11647577

Leonard, A. (2000). The viable system model and knowledge management. Kybernetes, 29(5/6), 710-715. https://doi.org/10.1108/03684920010333143

Lima, F. y De Souza, S. M. (2007). Gestão do conhecimento científico: proposta de um modelo conceitual com base em processos de comunicação científica. Ciência Da Informação, 36(1), 92-107. https://doi.org/10.1590/S0100-19652007000100007

Liyanage, S., Greenfield, P. F. \& Don, R. (1999). Towards a fourth generation R\&D management model-research networks in knowledge management. International Journal of Technology Management, 18(3/4), 372. https://doi.org/10.1504/IJTM.1999.002770 
Llamosa-Villalba, R., Carreño, L. T., Paéz, Q. A. M., Barajas, A. B. \& Sneyder, E. G. (2015). Enterprise architecture of Colombian Higher Education. En U. of T. SEE Educational Research and Methods Division, IEEE Computer Society, IEEE Education Society, New Mexico State University (Eds.), Frontiers in Education Conference, FIE. United States: Institute of Electrical and Electronics Engineers Inc.

Londoño-Patiño, J. A. y Acevedo-Álvarez, C. A. (2018). El aprendizaje organizacional (AO) y el desempeño empresarial bajo el enfoque de las capacidades dinámicas de aprendizaje. Revista CEA, 4(7), 103-118. https://doi.org/10.22430/24223182.762

Maher, P. E. \& Kourik, J. L. (2008). A knowledge management system for disseminating semi-structured information in a worldwide university. En PICMET '08 - 2008 Portland International Conference on Management of Engineering \& Technology (pp. 1936-1942). https://doi.org/10.1109/PICMET.2008.4599814

Marulanda-Valencia, F. Á. y Valencia-Arias, J. A. (2019). Evolución y tendencias investigativas en autoeficacia emprendedora: un análisis bibliométrico. Estudios Gerenciales, 35(151), 219-232. https://doi.org/10.18046/j.estger.2019.151.3277

Medina, V. H. Medina, L. M. \& Meza, J. J. (2018). Methodology for the Implementation of Knowledge Management Systems in the University. En Á. Rocha, H. Adeli, L. P. Reis, \& S. Costanzo (Eds.), 6th World Conference on Information Systems and Technologies, WorldCIST 2018 (pp. 161-170). https://doi.org/https://doi.org/10.1007/978-3-319-77703-0_16

Milla, L. E., Martelo, R. J. y Peña, M. (2018). Gestión del conocimiento para la difusión de producción intelectual en la educación universitaria. Saber, ciencia y libertad, 13(1), 290-303. https://doi.org/10.18041/2382-3240/saber.2018v13n1.2569

Mohammed, A. (2015). Knowledge Management Process in several organizations: Analytical Study of modeling and several processes. Procedia Computer Science, 65, 726-733.

Morgan, M. \& Llinàs, X. (2017). An academic knowledge management model: Multiple case studies in Peruvian Universities. En B.-M. J. Berbegal-Mirabent J., M. Mas-Machuca, R. Bastida, \& F. Marimon (Eds.), 18th European Conference on Knowledge Management, ECKM 2017 (pp. 730-741). Barcelona, Spain: Academic Conferences Limited.

Muñoz, D. E. \& Valencia, J. E. (2015). Knowledge management in organizations: A necessary encounter of digital platforms, communications, education and culture. Revista Lasallista de Investigacion, 12(2), 105-111.

Muñoz-García, A., Del Cioppo, J. \& Bucaram-Leverone, M. (2017). Ontology Model for the Knowledge Management in the Agricultural Teaching at the UAE. En International Conference on Technologies and Innovation (pp. 252-266). https://doi.org/10.1007/978-3-319-67283-0_19

Natek, S. \& Zwilling, M. (2014). Student data mining solution-knowledge management system related to higher education institutions. Expert Systems with Applications, 41(14), 6400-6407. https://doi.org/10.1016/j.eswa.2014.04.024

Numprasertchai, S. \& Poovarawan, Y. (2008). Improving university performance through ict based knowledge management system. International Journal of Innovation and Technology Management, 05(02), 167-178. https://doi.org/10.1142/S021987700800131X 
Oktavia, T., Spits, H. L. H., Adi, S., Prabowo, H. \& Supangkat, S. H. (2017). Knowledge management and social learning integration: A conceptual model for higher education. Far East Journal of Electronics and Communications, 16(4), 809-822. https://doi.org/10.17654/EC016040809

Palgrave. (2018). Communications in Computer and Information Science. Recuperado de https://www.palgrave.com/us/series/7899

Passailaigue, R. M., Márquez, F., Ortega, C. E. y Febles, A. (2017). Bases de una estrategia de gestión del conocimiento para la universidad inteligente de clase mundial. Espacios, 38(50), 13.

Raman, M., Ryan, T. \& Olfman, L. (2006). Knowledge Management System for Emergency Preparedness: An Action Research Study. Proceedings of the 39th Annual Hawaii International Conference on System Sciences (HICSS'06), 37b-37b. https://doi.org/10.1109/HICSS.2006.244

Rehn, C., Gornitzki, C., Larsson, A. \& Wadskog, D. (2014). Bibliometric handbook for Karolinska institutet. University library bibliometric team. Recuperado de https://kib.ki.se/sites/default/files/bibliometric_handbook_2014.pdf

Roohbakhsh, H., Gholizadeh, H. R. \& Javidi, T. J. K. (2017). Description and analysis of personal knowledge management skills among the staff of Ferdowsi University of Mashhad. Iranian Journal of Information Processing Management, 32(2), 375-395.

Shi, Y. \& Li, X. (2019). A bibliometric study on intelligent techniques of bankruptcy prediction for corporate firms. Heliyon, 5(12). https://doi.org/10.1016/j.heliyon.2019.e02997

Springer (s.f.). Lecture Notes in Computer Science (LNCS). Recuperado de https://www.springer.com/la/computer-science/lncs

Suroso, J. S., Sasmokon N. \& Elisabeth. (2017). The impact on knowledge management system's element to the performance in higher education. In N. K. K. Kwan, F. L. Wang, J. Shang y O. Au (Ed.), International Symposium on Educational Technology, ISET 2017 (pp. 180-183). https://doi.org/10.1109/ISET.2017.49

Teah, H. Y., Pee, L. G. \& Kankanhalli, A. (2006). Development and application of a General Knowledge Management Maturity Model. 10th Pacific Asia Conference on Information Systems: ICT and Innovation Economy, 401-416. Recuperado de https://www.scopus.com/record/display.uri?ei$d=2-s 2.0-80053514288 \&$ origin=inward\&txGid=03c8a7e1ef7c45735ec63c270bcb62b2

Thelwall, M. (2009). Bibliometrics and Citation Analysis: From the Science Citation Index to Cybermetrics. Library \& Information Science Research, 31(4), 268-269. https://doi.org/10.1016/J.LISR.2009.04.002

Tkachenko, E., Rogova, E. \& Karlik, A. (2017). Transformation of a Knowledge Management System in the Process of Educational Institutions' Merger. European Conference on Knowledge Management, 981-990. Recuperado de https://search.proquest.com/openview/575293445d8ac2aa6f2be 900602 bb1b1/1?pq-origsite=gscholar $\&$ cbl=1796412

UNESCO. (2009). Conferencia Mundial sobre la Educación Superior - 2009. La Nueva Dinámica de La Educación Superior y La Investigación Para El Cambio Social y El Desarrollo, 1-9. Recuperado de http://www.unesco.org/education/WCHE2009/comunicado_es.pdf 
Velázquez-Juárez, J. A., Valencia-Pérez, L. R. y Peña-Aguilar, J. M. (2016). El papel del modelo de la triple hélice como sistema de innovación para aumentar la rentabilidad en una Pyme comercializadora. Revista CEA, 2(3), 101-112. https://doi.org/10.22430/24223182.268

Waltman, L. \& Noyons, E. (2019). Bibliometrics for research management and research evaluation: A brief introduction. Recuperado de https://www.cwts.nl/pdf/CWTS_bibliometrics.pdf

Yankovskaya, V. V. \& Kukushkin, S. N. (May, 2019). The role of the high school in the "Triple Loop" model: SCBIN technologies. IOP Conference Series: Earth and Environmental Science, 274(1).

Yigzaw, S. T., Jormanainen, I. \& Tukiainen, M. (October, 2019). Trends in the role of ICT in Higher Education Knowledge Management Systems: A systematic literature review. En Proceedings of the Seventh International Conference on Technological Ecosystems for Enhancing Multiculturality (pp. 473-480). ACM. 